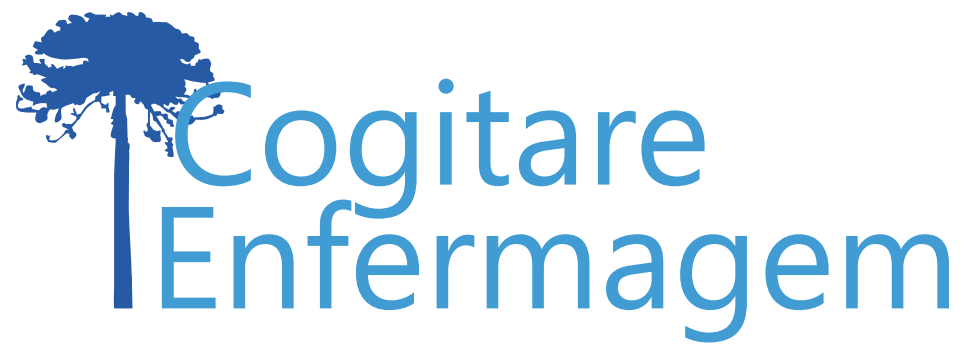

REVISÃO

\title{
FITOTERAPIA COMO INTERVENÇÃO EM SAÚDE DA MULHER: REVISÃO INTEGRATIVA DA LITERATURA
}

\author{
Maria Charlianne de Lima Pereira Silva ${ }^{1}$, Juan Carlos Alvarado Alcócer ${ }^{2}$, Leilane Barbosa de \\ Sousa ${ }^{3}$, Edmara Chaves Costa ${ }^{4}$, Olienaide Ribeiro de Oliveira Pinto ${ }^{5}$, Nathanael de Souza \\ Maciel $^{6}$, Francisco Jardsom Moura Luzia ${ }^{7}$
}

\section{RESUMO}

Objetivo: revisar as publicações científicas disponíveis na literatura que descrevem a fitoterapia como intervenção em saúde da mulher.

Método: revisão integrativa da literatura nas bases MEDLINE, CINAHL, Scopus e PUBMED. Adotouse a questão nordeadora: "Quais evidências disponíveis na literatura descrevem a utilização da fitoterapia como intervenção em saúde da mulher?". Os descritoresutilizadosforam: "Phytotherapy"; "Complementary Therapies"; "Women's Health". O processo de busca e levantamento dos dados ocorreu em novembro e dezembro de 2018, no município de Redenção-Ceará.

Resultados: foram analisados nove artigos, três relataram sobre plantas medicinais utilizadas no tratamento da menopausa, três sobre infertilidade, um sobre infecções ginecológicas e dois sobre distúrbios hormonais.

Conclusão: os estudos científicos acerca das plantas medicinais ainda são poucos, sendo fundamental a necessidade de mais estudos e pesquisas acerca da fitoterapia utilizada no tratamento das doenças ginecológicas.

DESCRITORES: Fitoterapia; Saúde da Mulher; Terapias complementares; Doenças dos Genitais Femininos; Plantas medicinais.

\section{COMO REFERENCIAR ESTE ARTIGO:}

Silva MC de LP, Alcócer JCA, Sousa LB de, Costa EC, Pinto OR de O, Maciel N de S, et al. Fitoterapia como intervenção em saúde da mulher: revisão integrativa da literatura. Cogitare enferm. [Internet]. 2020 [acesso em "colocar data de acesso, dia, mês abreviado e ano"]; 25. Disponível em: http://dx.doi.org/10.5380/ ce.v25i0.71158.

${ }^{1}$ Enfermeira. Mestre em Sociobiodiversidade e Tecnologias Sustentáveis. Docente do Instituto Centro de Ensino Tecnológico. Fortaleza, CE, Brasil.

${ }^{2}$ Físico. Doutor em Engenharia Elétrica. Docente da Universidade da Integração Internacional da Lusofonia Afro-Brasileira. Redenção, CE, Brasil.

${ }^{3}$ Enfermeira. Doutora em Enfermagem na Promoção da Saúde. Docente da Universidade da Integração Internacional da Lusofonia Afro-Brasileira. Redenção, CE, Brasil. (-)

${ }^{4}$ Médica Veterinária. Doutora em Ciências Veterinárias. Docente da Universidade da Integração Internacional da Lusofonia Afro-Brasileira. Redenção, CE, Brasil.

${ }^{5}$ Engenheira Agronômica. Doutora em Agronomia/Fitotecnia. Universidade da Integração Internacional da Lusofonia AfroBrasileira. Redenção, CE, Brasil. (2)

${ }^{6}$ Discente de Enfermagem. Universidade da Integração Internacional da Lusofonia Afro-Brasileira. Redenção, CE, Brasil. (2) ${ }^{7}$ Discente de Enfermagem. Universidade da Integração Internacional da Lusofonia Afro-Brasileira. Redenção, CE, Brasil. $\bigcirc$ 


\title{
PHYTOTHERAPY AS AN INTERVENTION IN WOMEN'S HEALTH: AN INTEGRATIVE LITERATURE REVIEW
}

\begin{abstract}
Objective: to review the scientific publications available in the literature that describe phytotherapy as intervention in women's health.

Method: an integrative literature review conducted in the MEDLINE, CINAHL, Scopus, and PUBMED databases. The following guiding question was adopted: "What evidence available in the literature describes the use of phytotherapy as intervention in women's health?" The descriptors used were the following: "Phytotherapy"; "Complementary Therapies"; "Women's Health". The process of surveying and gathering data took place in November and December 2018, in the city of Redenção, state of Ceará, Brazil.

Results: nine articles were analyzed, three of which discussed medicinal plants used to treat menopause, three infertility, one gynecological infections, and two hormonal disorders.

Conclusion: scientific studies on medicinal plants are still scarce, and the need for further studies and research on phytotherapy used in the treatment of gynecological diseases is essential.
\end{abstract}

DESCRIPTORS: Phytotherapy; Women's Health; Complementary Therapies; Diseases of the Female Genitals; Medicinal Plants.

\section{FITOTERAPIA COMO INTERVENCIÓN EN LA SALUD DE LA MUJER: UNA REVISIOON INTEGRADORA DE LA LITERATURA}

\author{
RESUMEN: \\ Objetivo: revisar las publicaciones científicas disponibles en la literatura que describen a la \\ fitoterapia como intervención en la salud de la mujer. \\ Método: revisión integradora de la literatura realizada en las bases de datos MEDLINE, \\ CINAHL, Scopus y PUBMED. Se adoptó la siguiente pregunta guía: "¿Qué evidencias se \\ ofrecen en la literatura que describan el uso de la fitoterapia como intervención en la salud \\ de la mujer?". Se utilizaron los siguientes descriptores: "Phytotherapy"; "Complementary \\ Therapies"; "Women's Health". El proceso de búsqueda y levantamiento de datos tuvo lugar \\ en noviembre y diciembre de 2018, en el municipio de Redenção-Ceará. \\ Resultados: se analizaron nueve artículos; tres de ellos analizaron las plantas medicinales \\ utilizadas en el tratamiento de la menopausia, tres la infertilidad, uno las infecciones \\ ginecológicas, y dos los trastornos hormonales. \\ Conclusión: la cantidad de estudios científicos acerca de las plantas medicinales todavía es \\ escasa, por lo que resulta fundamental realizar más estudios e investigaciones acerca de la \\ fitoterapia utilizada en el tratamiento de las enfermedades ginecológicas.
}

DESCRIPTORES: Fitoterapia; Salud de la Mujer; Terapias complementarias; Enfermedades de los genitales femeninos; Plantas medicinales. 
O uso de plantas para fins de saúde é datado desde os primórdios da humanidade(1). Em muitas situações, o uso de composições derivadas da natureza auxiliou no processo saúde-doença como forma de alívio para sinais e sintomas, como também para a cura de enfermidades, utilizando técnicas que são repassadas de modo empírico para outras gerações. No entanto, com o processo de industrialização e os avanços na indústria farmacêutica, o uso desse modelo de tratamento passou a ser preterido pela população(2).

No Brasil, a biodiversidade e a diversidade étnica e cultural permitiram um crescimento significativo do conhecimento quanto ao uso de medicamentos fitoterápicos e de plantas medicinais no processo terapêutico. Nessa perspectiva, com o propósito de estabelecer um controle governamental quanto ao uso desses produtos, em 2007 foi criada a Política Nacional de Plantas Medicinais e Fitoterápicos, garantindo, além da elaboração de pesquisas, a expansão do conhecimento para a população(3).

A Organização Mundial da Saúde (OMS) afirma que 80 a $85 \%$ da população, principalmente dos países em desenvolvimento como o Brasil, acredita no potencial resolutivo das plantas medicinais e as utiliza no tratamento de patologias diversas ${ }^{(4)}$. Porém, a ausência de informações por parte dos profissionais e o relato ineficaz por intermédio dos usuários acabaram mascarando reações adversas provocadas pelo uso desses insumos: 1728 casos de intoxicação pelo uso de fitoterápicos foram registrados no Brasil no ano de 2002, demandando o aprofundamento de pesquisas na área ${ }^{(5)}$.

Fatores importantes devem ser levados em consideração no que diz respeito ao aumento do uso de plantas medicinas no cotidiano. Com o elevado preço dos fármacos químicos disponíveis para comercialização e a dificuldade encontrada pela população ao acesso aos serviços de saúde, o profissional de enfermagem tem inserido, com base nas evidências científicas, produtos oriundos de plantas medicinais ${ }^{(6)}$.

As mulheres em idade fértil são compreendidas na faixa etária de 10 a $49 \operatorname{anos}^{(7)}$, representando $65 \%$ das mulheres brasileiras ${ }^{(8)}$. Esses números são levados em consideração na elaboração de estratégias de promoção da saúde sexual e reprodutiva, incluindo cada vez mais o uso de fitoterápicos e plantas medicinais no tratamento de problemas ginecológicos, ou mesmo durante a gravidez, o ciclo menstrual e a menopausa. Nesse sentido, à medida que profissionais da saúde, como enfermeiros, conhecem a fitoterapia, podem aplicá-la de maneira coerente nos serviços de saúde.

A aplicação das plantas medicinais e fitoterápicas é um possível meio de ampliação da área de trabalho dos profissionais de saúde que ainda estão pouco informados e preparados para lidar com esses recursos ${ }^{(2)}$. Salienta-se que a prescrição do fitoterápico a ser consumido pelo paciente é realizada por profissionais de saúde credenciados para definir o medicamento a ser usado(3).

Há uma variedade de plantas utilizadas para diversas finalidades, sendo 97 espécies associadas às questões de maternidade, 94 espécies ao ciclo menstrual e 243 espécies para o tratamento de outras condições ${ }^{(9)}$, o que revela uma pluralidade de composições e uso no processo terapêutico das afecções ginecológicas. Diante do exposto, o objetivo deste estudo é revisar as publicações científicas disponíveis na literatura que descrevem a fitoterapia como intervenção em saúde da mulher.

\section{MÉTODO}

O percurso metodológico adotado nessa revisão integrativa seguiu as etapas: 
identificação do tema e seleção da hipótese, estabelecimento de critérios para inclusão e exclusão de estudos, busca de literatura, categorização dos estudos, avaliação dos estudos incluídos na revisão integrativa, interpretação dos resultado e síntese da pesquisa ${ }^{(10)}$. Toda a pesquisa foi norteada pela indagação "Quais evidências disponíveis na literatura descrevem a utilização da fitoterapia como intervenção em saúde da mulher?".

O processo de busca e levantamento dos dados ocorreram em novembro e dezembro de 2018, no município de Redenção-CE. As bases de dados escolhidas foram: MEDLINE, Cummulative Index to Nursing and Allied Health Literature (CINAHL), Scopus e PUBMED.

Os descritores utilizados foram buscados no Medical Subject Headings (MeSH) e nos Descritores em Ciências da Saúde (DeCS), sendo eles: "Phytotherapy"; "Complementary Therapies"; "Women's Health", utilizando-se os operadores booleanos AND para a realização do cruzamento "Phytotherapy AND Complementary Therapies AND Women's Health". Com o intuito de ampliar a amostra, adotou-se uma segunda estratégia de combinação de descritores nas bases Medical Literature Analysis and Retrieval System Online (MEDLINE), Scopus e PUBMED: "Phytotherapy AND Women's Health".

Os critérios utilizados na inclusão dos artigos foram: artigos primários publicados nos últimos cinco anos (2014-2018), nos idiomas português, inglês e espanhol, que abordassem a pergunta norteadora. Além disso, os artigos deveriam estar disponíveis online nas plataformas e com acesso livre, de forma completa. Foram excluídos da pesquisa teses, dissertações, revisões de literaturas e editoriais, relatos de experiência, notas prévias e metanálises. A Figura 1 apresenta a síntese dos estudos para a revisão.
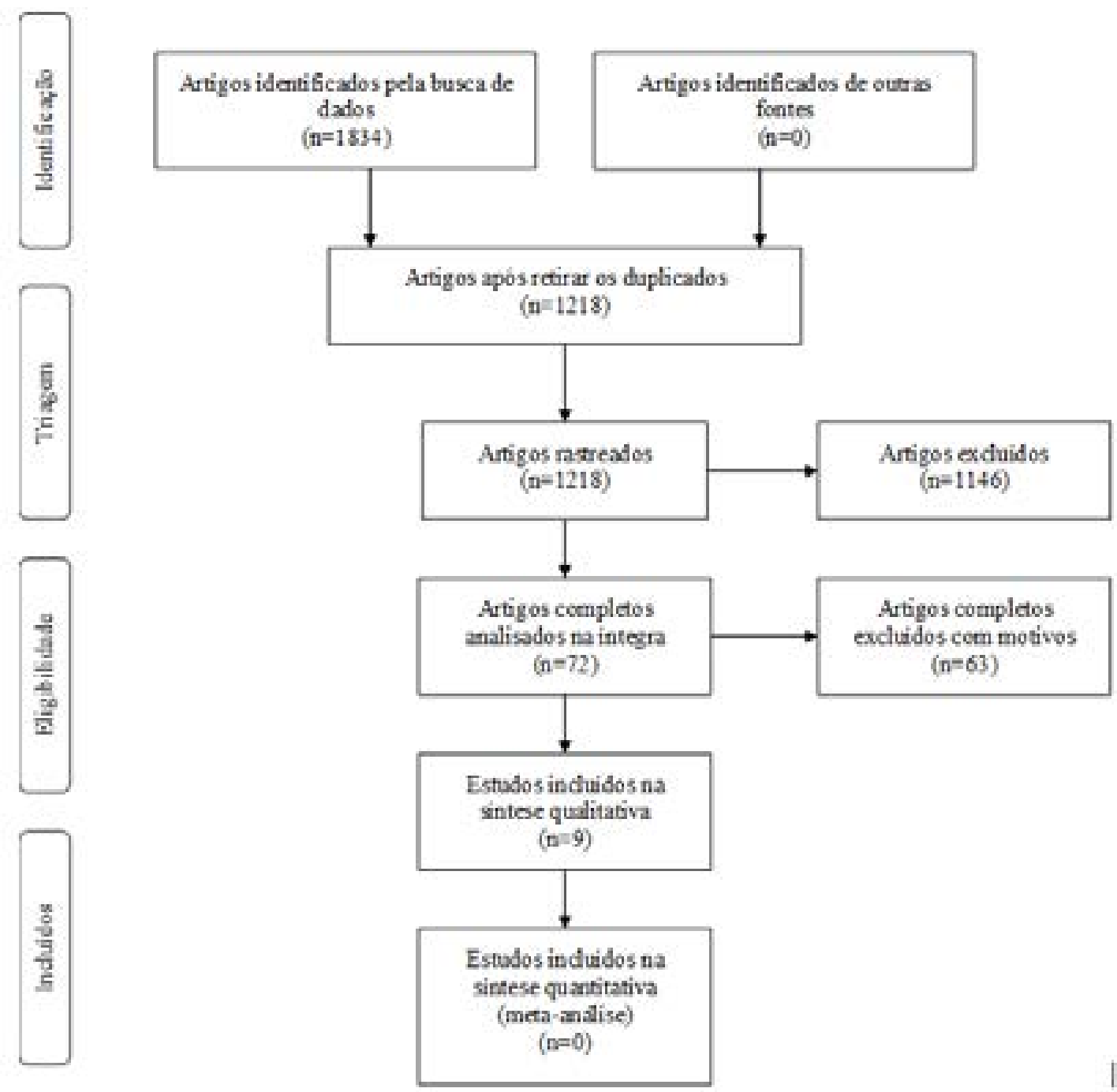

Figura 1 - Fluxograma referente a seleção dos artigos incluídos na revisão. Redenção, Ceará, Brasil, 2019 
Foram identificados 1834 artigos nas buscas empregadas nas bases de dados selecionadas, sendo excluídas 616 publicações duplicadas e, após a leitura de títulos, resumos e descritores, 72 artigos foram analisados na íntegra. Desses, 63 foram excluídos por não responderem à questão norteadora da pesquisa.

Após a leitura e seleção dos artigos, foram utilizados instrumentos criados pelos autores para extrair, de forma descritiva, informações dos artigos, contendo os seguintes tópicos: base de dados, periódico, título, autor, ano, objetivos, método e principais resultados. Ainda foram detalhados os níveis de evidência dos artigos encontrados, divididos em cinco níveis por tipos de estudo, destacando o nível um por se tratarem de estudos randomizados com um alto poder e tratamento estatístico ${ }^{(11)}$.

Os resultados foram organizados em quadros sinópticos e em categorias para discutir a importância das plantas medicinais no cuidado a saúde da mulher.

\section{RESULTADOS}

Foram analisados nove artigos, três relataram sobre plantas medicinais utilizadas no tratamento da menopausa, três sobre infertilidade, um sobre infecções ginecológicas e dois sobre distúrbios hormonais.

No Quadro 1, estão os dados gerais dos artigos encontrados. No Quadro 2, são apresentados de forma sintetizada os objetivos, nível de evidência, método de cada estudo e principais resultados presentes na literatura consultada. No Quadro 3, apresentam-se informações sobre as plantas medicinais mais citadas na literatura durante o levantamento da revisão integrativa.

Quadro 1 - Apresentação da síntese dos artigos incluídos na amostra total da revisão integrativa. Redenção, Ceará, Brasil, 2019 (continua)

\begin{tabular}{|l|l|l|l|l|}
\hline \multicolumn{2}{|l|}{$\begin{array}{l}\text { Base de } \\
\text { dados }\end{array}$} & Periódico & Título & Autor/ Ano \\
\hline 1 & CINAHL & $\begin{array}{l}\text { Phytotherapy } \\
\text { Research }\end{array}$ & $\begin{array}{l}\text { Estilo de vida combinado e fitoterapia em } \\
\text { mulheres obesas com síndrome do ovário } \\
\text { policístico (SOP): um estudo controlado } \\
\text { randomizado }\end{array}$ & $\begin{array}{l}\text { Arentz, et al. } \\
(2017)\end{array}$ \\
\hline 2 & SCOPUS & Medicine Baltimore & $\begin{array}{l}\text { Produtos à base de plantas chinesas para } \\
\text { infertilidade feminina em Taiwan: um estudo de } \\
\text { coorte baseado na população }\end{array}$ & $\begin{array}{l}\text { Hung, et al. } \\
(2016)\end{array}$ \\
\hline 3 & SCOPUS & $\begin{array}{l}\text { Journal of } \\
\text { Ethnopharmacology }\end{array}$ & $\begin{array}{l}\text { Plantas medicinais usadas no Lesoto para } \\
\text { tratamento de problemas reprodutivos e pós- } \\
\text { reprodutivos }\end{array}$ & $\begin{array}{l}\text { Moteetee e } \\
\text { Kose (2016) }\end{array}$ \\
\hline 4 & SCOPUS & $\begin{array}{l}\text { Journal of } \\
\text { Ethnopharmacology }\end{array}$ & $\begin{array}{l}\text { Estudo etnomedicobotânico do conhecimento } \\
\text { indígena sobre plantas medicinais utilizadas no } \\
\text { tratamento de problemas reprodutivos no distrito } \\
\text { de Nalbari, Assam, Índia }\end{array}$ & $\begin{array}{l}\text { Adhikari, et al. } \\
(2017)\end{array}$ \\
\hline 5 & SCOPUS & $\begin{array}{l}\text { Journal of } \\
\text { Complementary } \\
\text { and Integrative } \\
\text { Medicine }\end{array}$ & $\begin{array}{l}\text { Eficácia do Tamarindus indicus, Melia azadirach } \\
\text { e Santalum album no manejo sindrômico do } \\
\text { corrimento vaginal anormal: um estudo controlado } \\
\text { randomizado, simples-cego }\end{array}$ & $\begin{array}{l}\text { Bhat e Begum } \\
\text { (2017) }\end{array}$ \\
\hline
\end{tabular}




\begin{tabular}{|l|l|l|l|l|}
\hline 6 & SCOPUS & Maturitas & $\begin{array}{l}\text { Efeitos da ingestão de óleo de espinheiro } \\
\text { marítimo na atrofia vaginal em mulheres na pós- } \\
\text { menopausa: um estudo randomizado, duplo-cego, } \\
\text { controlado por placebo }\end{array}$ & $\begin{array}{l}\text { Larmo, et al. } \\
(2014)\end{array}$ \\
\hline 7 & SCOPUS & $\begin{array}{l}\text { BioMed Research } \\
\text { International }\end{array}$ & $\begin{array}{l}\text { Avaliação etnoginecológica de plantas medicinais } \\
\text { em sociedade tribal de Pashtun }\end{array}$ & $\begin{array}{l}\text { Adnan, et al. } \\
(2015)\end{array}$ \\
\hline 8 & SCOPUS & Holist Nurs Pract & $\begin{array}{l}\text { Os efeitos da linhaça sobre os sintomas da } \\
\text { menopausa e qualidade de vida }\end{array}$ & $\begin{array}{l}\text { Cetisli, et al. } \\
\text { (2015) }\end{array}$ \\
\hline 9 & SCOPUS & Climateric & $\begin{array}{l}\text { Extrato de Cimicifuga racemosa para aliviar } \\
\text { os sintomas da menopausa: um ensaio clínico } \\
\text { randomizado }\end{array}$ & $\begin{array}{l}\text { Tanmahasamut, } \\
\text { et al (2015) }\end{array}$ \\
\hline
\end{tabular}

Quadro 2 - Apresentação dos objetivos, nível de evidência, método e conclusão dos artigos incluídos na amostra final da revisão integrativa. Redenção, Ceará, Brasil, 2019 (continua)

\begin{tabular}{|c|c|c|c|c|}
\hline \multicolumn{2}{|c|}{ Objetivo } & \multirow{2}{*}{$\frac{\text { NE }}{\mathrm{I}}$} & \multirow{2}{*}{$\begin{array}{l}\text { Método } \\
\text { Ensaio controlado randomizado, } \\
\text { realizado em comunidades da } \\
\text { Austrália em mulheres de } 18 \text { a } 44 \text { anos } \\
\text { com SOP e excesso de peso. }\end{array}$} & \multirow{2}{*}{$\begin{array}{l}\text { Conclusão } \\
\text { Há uma melhor eficácia } \\
\text { no uso combinado de } \\
\text { medicamentos à base } \\
\text { de plantas em mulheres } \\
\text { com SOP. }\end{array}$} \\
\hline 1 & $\begin{array}{l}\text { Determinar a eficácia clínica } \\
\text { da combinação de um } \\
\text { tratamento fitoterápico com } \\
\text { uma intervenção no estilo de } \\
\text { vida para promover redução } \\
\text { na oligomenorreia em } \\
\text { mulheres com sobrepeso com } \\
\text { SOP. }\end{array}$ & & & \\
\hline 2 & $\begin{array}{l}\text { Identificar os fitoterápicos } \\
\text { chineses (CHPs) mais } \\
\text { utilizados para infertilidade } \\
\text { feminina em Taiwan. }\end{array}$ & IV & $\begin{array}{l}\text { Amostragem no banco de dados do } \\
\text { National Health Insurance Research } \\
\text { Database. A estatística descritiva e } \\
\text { análise de regressão logística múltipla } \\
\text { foram empregadas para estimar o } \\
\text { odds ratio ajustado (aOR) para uso da } \\
\text { MTC e fatores de risco potenciais. }\end{array}$ & $\begin{array}{l}\text { Combinações de } \\
\text { fórmulas de CHP ou } \\
\text { combinações de ervas } \\
\text { chinesas únicas foram } \\
\text { usadas para tratar a } \\
\text { infertilidade feminina, } \\
\text { sendo necessário outros } \\
\text { estudos. }\end{array}$ \\
\hline 3 & $\begin{array}{l}\text { Documentar as plantas usadas } \\
\text { pelo Basotho masculino } \\
\text { e feminino (residentes no } \\
\text { Lesoto) para o tratamento } \\
\text { de doenças reprodutivas e } \\
\text { utilização em outras culturas } \\
\text { da África Austral. }\end{array}$ & V & $\begin{array}{l}\text { Entrevistas de médicos tradicionais } \\
\text { no distrito de Maseru, experiências } \\
\text { do próprio autor e observações do } \\
\text { Nek District de Qacha. Pesquisa } \\
\text { bibliográfica em livros, dados não } \\
\text { publicados e bancos de dados } \\
\text { eletrônicos. }\end{array}$ & $\begin{array}{l}\text { A marioria das espécies } \\
\text { (13) são utilizadas } \\
\text { no tratamento } \\
\text { de infertilidade. } \\
\text { Contudo, os efeitos } \\
\text { farmacológicos, } \\
\text { compostos ativos e } \\
\text { toxicologia de muitas } \\
\text { destas plantas ainda } \\
\text { não são conhecidos. }\end{array}$ \\
\hline 4 & $\begin{array}{l}\text { Documentar o nome local } \\
\text { das plantas medicinais usadas } \\
\text { por homens e mulheres do } \\
\text { conhecimento indígena para } \\
\text { o tratamento de doenças } \\
\text { reprodutivas, além de } \\
\text { explorar seus metabólitos } \\
\text { secundários neles presentes. }\end{array}$ & V & $\begin{array}{l}\text { Entrevistas aos médicos tradicionais } \\
\text { em três aldeias. Foram analisados } \\
\text { sistemáticamente porcentagem de } \\
\text { nível de fidelidade, percentual de } \\
\text { ranking de preferência e pontuação } \\
\text { de formulação. Utilizou-se também } \\
\text { bancos de dados eletrônicos. }\end{array}$ & $\begin{array}{l}\text { Os dados podem ser o } \\
\text { recurso mais importante } \\
\text { para a nova descoberta } \\
\text { de muitos princípios } \\
\text { bioativos. }\end{array}$ \\
\hline
\end{tabular}




\begin{tabular}{|c|c|c|c|c|}
\hline 5 & $\begin{array}{l}\text { Avaliar a eficácia da surfaid } \\
\text { de sândalo, maghze tukhme } \\
\text { bakayin e khaste tamar hindi } \\
\text { no manejo sindrômico do } \\
\text { corrimento vaginal. }\end{array}$ & II & $\begin{array}{l}\text { Estudo randomizado, simples cego, } \\
\text { controlado padrão. O grupo de } \\
\text { teste recebeu } 10 \mathrm{~g} \text { do fármaco de } \\
\text { teste durante } 21 \text { dias, enquanto o } \\
\text { grupo controle recebeu dose única } \\
\text { de fármaco padrão para ambos os } \\
\text { parceiros. } O \text { escore de sintomas } \\
\text { vaginais foi utilizado para avaliar a } \\
\text { alta e as queixas associadas. Escala } \\
\text { visual análoga foi utilizada para avaliar } \\
\text { lombalgia e dor abdominal baixa. }\end{array}$ & $\begin{array}{l}\text { A formulação pode } \\
\text { efetivamente aliviar a } \\
\text { doença com sintomas } \\
\text { associados sem efeitos } \\
\text { colaterais. Pode ser } \\
\text { usado no tratamento } \\
\text { sindrômico do } \\
\text { corrimento vaginal. }\end{array}$ \\
\hline 6 & $\begin{array}{l}\text { Investigar os efeitos da } \\
\text { suplementação com óleo de } \\
\text { espinheiro-mar sobre a atrofia } \\
\text { vaginal. }\end{array}$ & I & $\begin{array}{l}116 \text { mulheres na pós-menopausa } \\
\text { foram randomizados em estudo } \\
\text { duplo-cego controlado por placebo. } \\
\text { Durou } 3 \text { meses, com } 3 \mathrm{~g} \text { de óleo de } \\
\text { espinheiro marítimo (SB) ou óleo } \\
\text { placebo diariamente. No início e final, } \\
\text { os fatores de saúde vaginal foram } \\
\text { avaliados por um ginecologista, o pH } \\
\text { vaginal e a umidade foram medidos } \\
\text { e o índice de saúde vaginal foi } \\
\text { calculado. Os sintomas de atrofia e } \\
\text { menopausa foram avaliados. Amostras } \\
\text { de soro foram coletadas para a } \\
\text { análise de lipídios circulantes, enzimas } \\
\text { hepáticas e proteína C-reativa. }\end{array}$ & $\begin{array}{l}\text { De acordo com seus } \\
\text { efeitos sobre a saúde } \\
\text { vaginal, o óleo foi } \\
\text { indicado como uma } \\
\text { alternativa potencial } \\
\text { para as mulheres } \\
\text { que não podem usar } \\
\text { o tratamento com } \\
\text { estrogênio para a } \\
\text { atrofia vaginal }\end{array}$ \\
\hline 7 & $\begin{array}{l}\text { O estudo foi projetado para } \\
\text { documentar o conhecimento } \\
\text { etnoginecnológico detalhado } \\
\text { de regiões remotas } \\
\text { selecionadas da tribo de } \\
\text { Pashtun no noroeste do } \\
\text { Paquistão }\end{array}$ & IV & $\begin{array}{l}\text { O estudo foi realizado em seis áreas } \\
\text { remotas da província de Khyber } \\
\text { Pakhtunkhwa (KPK), no Paquistão. Foi } \\
\text { documentado de janeiro a junho de } \\
2014 \text {. O consenso dos informantes } \\
\text { foi o método para destacar as plantas } \\
\text { amplamente utilizadas para doenças } \\
\text { específicas. }\end{array}$ & $\begin{array}{l}\text { Plantas com altos } \\
\text { valores de Fator } \\
\text { de Consenso dos } \\
\text { Informantes (FIC) devem } \\
\text { ser checadas para sua } \\
\text { validação in vitro e in } \\
\text { vivo. As meninas devem } \\
\text { ser educadas sobre a } \\
\text { importância das práticas } \\
\text { etnogno-ecológicas } \\
\text { para conservar esse } \\
\text { valioso conhecimento. }\end{array}$ \\
\hline 8 & $\begin{array}{l}\text { Analisar os efeitos da linhaça } \\
\text { sobre sintomas da menopausa } \\
\text { e qualidade de vida } \\
\text { durante o período da } \\
\text { menopausa }\end{array}$ & III & $\begin{array}{l}\text { Esta pesquisa experimental foi } \\
\text { planejada para ser composta de } \\
\text { pré-testes, pós-testes e um grupo } \\
\text { de controle de mulheres que usaram } \\
\text { linhaça e receberam treinamento e } \\
\text { terapia de reposição hormonal (TRH). }\end{array}$ & $\begin{array}{l}\text { Houve diminuição dos } \\
\text { sintomas da menopausa } \\
\text { e melhora na vida entre } \\
\text { as mulheres que usaram } \\
\text { linhaça durante três } \\
\text { meses. } \\
\text { Os resultados servem } \\
\text { como referência para } \\
\text { os prestadores de } \\
\text { cuidados clínicos. }\end{array}$ \\
\hline 9 & $\begin{array}{l}\text { Avaliar a eficácia do extrato } \\
\text { de cohosh preto } 40 \text { mg/dia } \\
\text { para alívio de sintomas da } \\
\text { menopausa moderados a } \\
\text { graves e melhorar a qualidade } \\
\text { de vida em mulheres } \\
\text { tailandesas }\end{array}$ & II & $\begin{array}{l}\text { Ensaio clínico randomizado, duplo- } \\
\text { cego, controlado por placebo. } \\
\text { Foi conduzido em uma clínica } \\
\text { de menopausa de um hospital } \\
\text { universitário durante } 2011-2013 . \\
\text { Os participantes foram mulheres } \\
\text { tailandesas peri ou pós-menopausa } \\
\text { com pelo menos } 40 \text { anos de idade, } \\
\text { que apresentam sintomas moderados } \\
\text { a graves do índice de Kupperman (KI). }\end{array}$ & $\begin{array}{l}\text { O extrato de cohosh } \\
\text { preto de } 40 \text { mg/dia não } \\
\text { é superior a um placebo } \\
\text { para alívio de sintomas } \\
\text { moderados a graves da } \\
\text { menopausa ou melhorar } \\
\text { os escores de qualidade } \\
\text { de vida em mulheres } \\
\text { tailandesas }\end{array}$ \\
\hline
\end{tabular}


Quadro 3 - Apresentação das plantas medicinais citadas nos estudos da revisão integrativa. Redenção, Ceará, Brasil, 2019

\begin{tabular}{|c|c|c|c|c|}
\hline \multicolumn{2}{|c|}{ Nome científico } & \multirow{2}{*}{$\begin{array}{l}\text { Autor/ Ano } \\
\text { Larmo, } \\
\text { et al. (2014) }\end{array}$} & \multirow{2}{*}{$\begin{array}{l}\text { Modo de } \\
\text { uso } \\
\text { Óleo }\end{array}$} & \multirow{2}{*}{$\begin{array}{l}\text { Problemas ginecológicos citados } \\
\text { Inflamações nos órgãos genitais } \\
\text { e no útero; alivia os sintomas } \\
\text { associados à secura das } \\
\text { membranas das mucosas genitais } \\
\text { (atrofia vaginal) } \\
\end{array}$} \\
\hline 1 & $\begin{array}{l}\text { Óleo de espinheiro marítimo } \\
\text { (Hippophaë rhamnoides) }\end{array}$ & & & \\
\hline 2 & Cimicifuga racemosa (black cohosh) & $\begin{array}{l}\text { Tanmahas, } \\
\text { et al. (2015) }\end{array}$ & Decocções & $\begin{array}{l}\text { Sintomas da menopausa de } \\
\text { moderada a grave }\end{array}$ \\
\hline 3 & $\begin{array}{l}\text { Dang-Gui-Sha-Yao-San, Wen-Jing- } \\
\text { Tang, Jia- Wei-Xiao-Yao-San, Zou- } \\
\text { Gui-Wan, You-Gui- Wan, Leonurus } \\
\text { japonicus, Fructus ligustri lucidi, } \\
\text { Cyperus rotundus L., Dipsacus } \\
\text { asper Wall, Semen Cuscutae }\end{array}$ & $\begin{array}{l}\text { Hung, et al. } \\
\text { (2016) }\end{array}$ & Decocções & $\begin{array}{l}\text { Melhora a disfunção endócrina } \\
\text { ovariana, infertilidade feminina, } \\
\text { tratamento de perturbações da } \\
\text { ovulação, suprime a contração do } \\
\text { músculo liso do útero e atua como } \\
\text { efeito antioxidante no útero }\end{array}$ \\
\hline 4 & Gunnera perpensa & $\begin{array}{l}\text { Hung, et al. } \\
\text { (2016) }\end{array}$ & Decocções & $\begin{array}{l}\text { Doenças reprodutivas maternas } \\
\text { (como dismenorreia), afecções, } \\
\text { atividade anti-inflamatória, dores } \\
\text { menstruais e tonificação do útero }\end{array}$ \\
\hline 5 & Withania somnifera & $\begin{array}{l}\text { Hung, et al. } \\
(2016)\end{array}$ & $\begin{array}{l}\text { Decocções } \\
\text { e sucos }\end{array}$ & Infertilidade \\
\hline 6 & Scabiosa columbaria & $\begin{array}{l}\text { Hung, et al. } \\
(2016)\end{array}$ & Decocções & $\begin{array}{l}\text { Tratamento da infertilidade, } \\
\text { dismenorreia, tratamento de } \\
\text { distúrbios uterinos }\end{array}$ \\
\hline 7 & $\begin{array}{l}\text { Malva parviflora; Xysmalobium } \\
\text { undulatum; Dicoma anomala; B. } \\
\text { setifera }\end{array}$ & $\begin{array}{l}\text { Adhikari, et } \\
\text { al. (2017) }\end{array}$ & Decocções & $\begin{array}{l}\text { Tratamento de distúrbios uterinos } \\
\text { não especificados e leucorreia }\end{array}$ \\
\hline
\end{tabular}

A partir da leitura na íntegra dos artigos, os estudos selecionados foram agrupados para discussão conforme à sua temática principal, assim especificados: Menopausa; Infertilidade; Infecções ginecológicas; Distúrbios hormonais.

\section{DISCUSSÃO}

\section{Menopausa}

A menopausa é um mecanismo natural e fisiológico para as mulheres. Para aumentar a qualidade de vida em termos de saúde física, mental e social, estas mulheres precisam de informações, apoio e acompanhamento regular de um profissional de saúde ${ }^{(12)}$.

A transição da menopausa ou perimenopausa é o estágio de vida reprodutiva das mulheres que começa alguns anos antes da menopausa, quando os ovários gradualmente começam a produzir menos estrogênios, em que muitas mulheres apresentam sintomas como afrontamentos, períodos irregulares e dificuldade de dormir ${ }^{(13)}$. 
Em estudo(12), os estrogênios de ervas inseridos na dieta das mulheres aumentaram o nível de estrogênio, evitando os sintomas causados pela menopausa. Os fitoestrógenos são produtos químicos que podem agir como um estrogênio natural no corpo humano. Não há síntese ou depósito desses compostos no corpo, e sua única fonte é uma dieta intensiva de ervas contendo esses compostos. Eles podem ser encontrados em grãos, vegetais e frutas, sendo suas fontes mais conhecidas e enriquecidas de fitoestrógenos a linhaça e a soja.

As mulheres menopausadas que receberam uma dieta rica em semente de linhaça e soja por 12 semanas apresentaram uma diminuição significativa nos sintomas da menopausa, como ondas de calor e secura vaginal, sendo preferível o uso de linhaça por causa da facilidade em seu uso e ausência de efeitos colaterais, como na TRH ${ }^{(12)}$.

A atrofia vaginal, o afinamento e a menor lubrificação da mucosa vaginal estão associados a níveis reduzidos de estrogênio na menopausa. O tecido atrófico é propenso a inflamação, hemorragias e ulceração. Em estudo ${ }^{(14)}$ foi destacado o uso do óleo de espinheiro marítimo, tradicionamente utilizado na Ásia Central no tratamento de inflamações nos órgãos genitais e no útero. A ingestão do óleo demonstrou, em estudos clínicos, efeitos benéficos sobre lipídios e lipoproteínas séricas, olho seco, marcadores de inflamação endotelial e agregação plaquetária.

A utilização da fitoterapia nos problemas ginecológicos também destacou o óleo de neroli (Citrus aurantium L. var. amara), um composto fitoterápico que, além de minimizar os sintomas da menopausa, também eleva o desejo sexual e diminui a pressão arterial na pósmenopausa. O óleo pode ser ser considerado uma intervenção inovadora e útil na redução do estresse e melhora no sistema endócrino ${ }^{(15)}$.

A planta Cimicifuga racemosa foi um dos fitoterápicos usados para aliviar os sintomas da menopausa, especialmente na América do Norte. Seu rizoma e o extrato de sua raiz contêm muitos princípios ativos, incluindo glicosídeos triterpênicos e ácidos fenólicos ${ }^{(16)}$. Ressaltando seu uso na melhora na qualidade de vida das mulheres tailandesas com sintomas de menopausa moderada a grave.

Aproximadamente $30 \%$ das participantes relataram efeitos colaterais, a maioria dos quais foram leves e autolimitados, sem tratamento ou término do estudo, uma vez que a medicação não demonstrou hepatotoxicidade nas participantes. $O$ estudo foi conduzido com o extrato de $1,28 \mathrm{mg}$ de princípios ativos por 12 semanas $^{(16)}$.

\section{Infertilidade}

A infertilidade feminina é definida como a incapacidade de engravidar em idade fértil, mesmo que haja relação sexual frequente e desprotegida há pelo menos um a quatro anos. As principais causas são distúrbios ovulatórios, endometriose, adesão pélvica, adquiridos (miomas), bloqueio tubário e outras anormalidades uterinas e nas trompas, indicandose o uso da medicina tradicional chinesa (MTC), com adoção de Terapias Integrativas e Complementares ${ }^{(17)}$.

Contribuindo com os autores acima, um estudo(18) registrou 32 espécies de plantas que foram utilizadas para o tratamento de vários problemas ginecológicos e obstétricos. Foram destacados dismenorreia, infertilidade e a menorragia. A dismenorreia teve 21 espécies de plantas, a infertilidade 14 e menorragia 10. Bridelia catartica foi a espécie vegetal mais citada pelas mulheres para o tratamento de problemas ginecológicos e obstétricos. Os modos de preparações das plantas foram, em sua maioria, misturas administradas oralmente.

As principais ervas descritas para as mulheres com infertilidade foram a Dang-GuiSha-Yao-San, uma fórmula fitoterápica mais comumente prescrita $(17,25 \%)$, seguido por Wen-Jing-Tang (16,35\%), Jia-Wei-Xiao-Yao-San (14,85\%), Zou-Gui-Wan (14,57\%) e GuiPi-Wan $(9,97 \%)$. O sêmen Cuscutae $(27,40 \%)$ foi também comum na prescrição de CHP, 
seguida de Leonurus japonicus (13,55\%), Fructus ligustri lucidi $(13,43)$, Cyperus rotundus L. $(12,13 \%)$ e Dipsacus asper Wall $(11,71 \%)$, destacando também, que, além de visitar o ginecologista, a maioria das pacientes $(96,17 \%)$ também procuram tratamento na MTC ${ }^{(17)}$.

Problemas reprodutivos como esterilidade, problemas mestruais e distúrbios uterinos podem ser tratadas com cerca de 87 diferentes espécies botânicas. A Gunnera perpensa é a mais utilizada para combater uma série de doenças reprodutivas, tendo outros usos farmacológicas, como analgésico, anti-inflamatório, antiespasmódico e uterotônico, o que poderia explicar seu uso para dores menstruais e tonificação do útero(19).

Outros autores ${ }^{(20)}$ recomendaram o Hibiscus rosa-sinensis, usado para curar a menstruação irregular, o suco de Cynodon dactylon, utilizado contra o sangramento uterino, as raízes de Abroma augusta contra gonorreia e os frutos de Bombax ceiba para a cura da infertilidade feminina e outros distúrbios ginecológicos. Algumas espécies de plantas raras, mas medicinalmente importantes como Andrographis paniculata, também foram relatadas no tratamento do transtorno sexual e vaginite.

\section{Infecções ginecológicas}

A infecção vaginal é uma das infecções ginecológicas mais comuns, sendo motivo das mulheres procurarem atendimento com a ginecologista. As infecções do trato reprodutivo tornaram-se uma epidemia silenciosa que assola a vida das mulheres e representam um problema nos países em desenvolvimento, sendo mais recorrentes em mulheres com idade entre 25 e 35 anos. Em $96 \%$ das pacientes o pH apresentou-se elevado, sendo um indicador sensível para Gardnerella vaginallis(21).

Relata-se a vaginite como uma síndrome causada principalmente por três grupos de patógenos microbianos (bactérias, leveduras e protozoários), sendo responsável por $90 \%$ das doenças. Salienta-se as propriedades adstringentes das plantas Sândalo, Tamarindo e Bakayin na melhoria do corrimento vaginal(21).

Outros estudos confirmam o uso das plantas medicinais no cuidado à saúde da mulher. As espécies Alternanthera brasiliana L, Alternanthera sp, Apium graveolens L, Artemisia vulgaris L, Tagetes minuta L, Impatiens balsamina L, Bryophyllum pinnatum L e Piptadenia gonoacantha foram as mais indicadas para o tratamento das doenças ginecológicas, como infecções uterinas, cólicas menstruais e inflamação dos ovários ${ }^{(22)}$.

O capim-santo (Cymbopogon citratus), a erva-cidreira (Lippia alba) e a courama (Kalanchoe brasiliensis) tambem são apontadas como plantas medicinais utilizadas pelas mulheres no tratamento de pequenas crises de cólicas uterinas, inflamações ovarianas e uterinas $^{(23)}$.

\section{Distúrbios hormonais}

A síndrome dos ovários policísticos (SOP) é um distúrbio reprodutivo e endócrino complexo, caracterizado por irregularidades menstruais (oligomenorreia/amenorreia), hiperandrogenismo, ovários policísticos, distúrbios metabólicos e psicológicos, afetando até $18 \%$ das mulheres em idade reprodutiva. Aproximadamente duas em cada cinco mulheres com SOP relatam o uso de fitoterapia, porque estes medicamentos à base de plantas contêm substâncias químicas biologicamente ativas que podem alterar a endocrinologia reprodutiva em mulheres com SOP(24).

O uso das plantas medicinais no tratamento das doenças ginecológicas, estudadas pelos autores(25) evidenciam que a maioria das plantas (19) foram utilizadas no controle de complicações durante a menstruação, enquanto outras (11) foram utilizadas no tratamento de gonorreia e gravidez. Destacou-se também nesse estudo as partes das plantas utilizadas pelas mulheres: plantas inteiras (33\%) e folhas (31\%) para várias preparações etnomedicinais na área da ginecologia. As mulheres das regiões do Paquistão usam principalmente ervas 
(59\%) para a preparação de etnomedicamentos, seguidas de árvores (26,9\%).

$\mathrm{Na}$ maioria das áreas remotas, as ervas medicinais são os principais ingredientes dos medicamentos locais e considerados a primeira escolha no tratamento das doenças ginecológicas ${ }^{(25)}$. As plantas Justicia adhatoda, Schinus molle, Convolvulus arvensis, Cyperus rotundus e Hypericum perforatum foram identificadas como sendo eficazes no alívio das complicações menstruais, enquanto a Melia azadirachta e Solanum surattense foram administradas no tratamento da gonorreia(25).

Dados etnográficos mostraram que a maioria das mulheres entrevistadas (44\%) tinham entre 61 e 70 anos, indicando claramente que o conhecimento tradicional é restrito a pessoas idosas dessas regiões, devido ao menor interesse da geração mais nova ${ }^{(25)}$.

Em outro estudo(26), mulheres com SOP relatam os benefícios do chá de manjerona, apontando melhora na sensibilidade à insulina e redução nos níveis de andrógenos. Entretanto, mais pesquisas são necessárias para confirmar esses resultados e investigar os componentes ativos e os mecanismos que contribuem para esses potenciais efeitos benéficos da manjerona.

\section{CONCLUSÃO}

Os achados deste estudo indicam um baixo número de artigos que abordam fitoterapia na intervenção em saúde da mulher. É importante salientar que as plantas medicinais podem ser utilizadas para diminuição dos sinais da menopausa e tratamento da infertilidade, de infecções ginecológicas e de distúrbios hormonais.

A síntese do conhecimento indicou a necessidade de intensificar esforços para o desenvolvimento de mais pesquisas com fitoterapia na intervenção da saúde da mulher no tratamento das doenças ginecológicas, sem desconsiderar os conhecimentos tradicionais acerca das plantas medicinais que são transmitidos entre gerações.

Torna-se imprescindível a participação dos profissionais de saúde na orientação da população em relação ao uso da fitoterapia como intervenção em ginecologia, tendo em vista a peculiaridade de cada planta e sua utilização adequada. Ademais, os benefícios do uso das plantas medicinais para a população feminina são redução de custos com saúde, promoção de saúde e prevenção de patologias, com eficácia comprovada cientificamente e junção do conhecimento empírico.

\section{REFERÊNCIAS}

1. Lima LO, Gomes EC. Alimento ou medicamento? Espécies vegetais frente à legislação brasileira. Rev. bras. plantas med. [Internet]. 2014 [acesso em 05 nov 2019]; 16(3). Disponível em: http://www.scielo.br/ scielo.php?script=sci arttext\&pid=S1516-05722014000700020.

2. Bruning MCR, Mosegui GBG, Vianna CM de M. A utilização da fitoterapia e de plantas medicinais em unidades básicas de saúde nos municípios de Cascavel e Foz do Iguaçu-Paraná: a visão dos profissionais de saúde. Ciênc. saúde colet [Internet]. 2012 [acesso em 05 nov 2019]; 17(10). Disponível em: http:// dx.doi.org/10.1590/S1413-81232012001000017.

3. Ministério da Saúde (BR). Secretaria de Ciência, Tecnologia e Insumos Estratégicos. Departamento de Assistência Farmacêutica. Política e Programa Nacional de Plantas Medicinais e Fitoterápicos. Brasília:

Ministério da Saúde; 2016. 
4. Souza CMP, Brandão DO, Silva MSP, Palmeira AC, Simões MOS, Medeiros ACD. Utilização de plantas medicinais com atividade antimicrobiana por usuários do serviço público de saúde em Campina GrandeParaíba. Rev. bras. plantas med. [Internet]. 2013 [acesso em 05 nov 2019]; 15(2). Disponível em: http:// dx.doi.org/10.1590/S1516-05722013000200004.

5. Silveira PF da, Bandeira MAM, Arrais PSD. Farmacovigilância e reações adversas às plantas medicinais e fitoterápicos: uma realidade. Rev. bras. farmacogn. [Internet] 2008. [acesso em 05 nov. 2019]; 18(4). Disponível em: http://dx.doi.org/10.1590/S0102-695X2008000400021.

6. Badke MR, Budó M de LD, Alvim NAT, Zanetti GD, Heisler EV. Popular knowledge and practices regarding healthcare using medicinal plants. Texto contexto-enferm [Internet] 2012. [acesso em 05 nov. 2019]; 21(2). Disponível em: http://dx.doi.org/10.1590/S0104-07072012000200014.

7. Ministério da Saúde (BR). Secretaria de Atenção à Saúde. Departamento de Ações Programáticas Estratégicas. Política nacional de atenção integral à saúde da mulher: princípios e diretrizes. Brasília: Ministério da Saúde; 2004.

8. IBGE. Instituto Brasileiro de Geografia e Estatística. Censo demográfico de 2010. [Internet]. 2010. [acesso em 20 maio 2019]. Disponível em: http://www.ibge.gov.br

9. Yazbek PB, Tezoto J, Cassas F, Rodrigues E. Plants used during maternity, menstrual cycle and other women's health conditions among Brazilian cultures. J Ethnopharmacol [Internet] 2016. [acesso em 05 nov. 2019]; 179(1). Disponível em: http://dx.doi.org/10.1016/j.jep.2015.12.054.

10. Mendes KDS, Silveira RC de CP, Galvão CM. Revisão integrativa: método de pesquisa para a incorporação de evidências na saúde e na enfermagem. Texto contexto-enferm. [Internet] 2008. [acesso em 05 nov. 2019]; 17(4). Disponível em: http://dx.doi.org/10.1590/S0104-07072008000400018.

11. Pereira AL, Bachion MM. Atualidades em revisão sistemática de literatura, critérios de força e grau de recomendação de evidência. Rev. Gaúcha Enferm. [Internet] 2006 [acesso em 05 nov. 2019]; 27(4). Disponível em: https://seer.ufrgs.br/RevistaGauchadeEnfermagem/article/view/4633.

12. Cetisli NE, Saruhan A, Kivcak B. The effects of flaxseed on menopausal symptoms and quality of life. Holist Nurs Pract. [Internet] 2015. [acesso em 05 nov. 2019]; 29(3). Disponível em: https://pubmed.ncbi. nlm.nih.gov/25882265/.

13. D'Anna R, Santamaria A, Giorgianni G, Vaiarelli A, Gullo G, Bari FD, et al. Myo-inositol and melatonin in the menopausal transition. Gynecol Endocrinol [Internet] 2017. [acesso em 05 nov. 2019]; 33(4).

Disponível em: https://doi.org/10.1080/09513590.2016.1254613.

14. Larmo PS, Yang B, Hyssala J, Kallio HP, Erkkola R. Effects of sea buckthorn oil intake on vaginal atrophy in postmenopausal women: a randomized, double-blind, placebo-controlled study. Maturitas. [Internet] 2014. [acesso em 05 nov. 2019]; 79(3). Disponível em: https://doi.org/10.1016/j. maturitas.2014.07.010.

15. Choi SY, Kamg P, Lee HS, Seol GE. Effects of inhalation of essential oil of citrus aurantium L. var. amaraon Menopausal Symptoms, Stress, and Estrogen in Postmenopausal Women: A Randomized Controlled Trial. Evid-based Compl Alt Med [Internet] 2014. [acesso em 05 nov. 2019]; (ID796518). Disponível em: https://doi.org/10.1155/2014/796518.

16. Tanmahasamut P, Vichinsartvichai P, Rattanachaiyanont M, Techatraisak K, Dangrat C, Sardod P. Cimicifuga racemosa extract for relieving menopausal symptoms: a randomized controlled trial. Climacteric. [Internet] 2014. [acesso em 05 nov. 2019]; 18(1). Disponível em: https://doi.org/10.3109/1369 7137.2014.933410.

17. Hung YC, Kao CW, Lin CC, Liao YN, Wu BY, Hung IL, et al. Chinese herbal products for female infertility in Taiwan: a population-based cohort study. Medicine (Baltimore). [Internet] 2016. [acesso em 05 nov. 2019]; 95(11). Disponível em: https://www.ncbi.nlm.nih.gov/pubmed/26986137.

18. Wet $\mathrm{H}$ de, Ngubane SC. Traditional herbal remedies used by women in a rural community in northern Maputaland (South Africa) for the treatment of gynaecology and obstetric complaints. S 
Afr J Bot. [Internet] 2014. [acesso em 05 nov. 2019]; 94(1) Disponível em: https://doi.org/10.1016/j. sajb.2014.06.009.

19. Moteetee A, Kose LS. Medicinal plants used in Lesotho for treatment of reproductive and post reproductive problems. J. Ethnopharmacol. [Internet] 2016. [acesso em 05 nov. 2019]; 194. Disponível em: http://dx.doi.org/10.1016/j.jep.2016.10.062.

20. Adhikari PP, Talukdar S, Borah A. Ethnomedicobotanical study of indigenous knowledge on medicinal plants used for the treatment of reproductive problems in Nalbari district, Assam, India. J. Ethnopharmacol. [Internet] 2018. [acesso em 05 nov. 2019]; 210. Disponível em: http://dx.doi. org/10.1016/j.jep.2017.07.024.

21. Bhat TA, Begum W. Efficacy of Tamarindus indicus, Melia azadirach and Santalum album in syndromic management of abnormal vaginal discharge: a single-blind randomised controlled trial. J Compl Integr Med [Internet] 2017. [acesso em 05 nov. 2019]; 15(2). Disponível em: http://dx.doi.org/10.1515/jcim2015-0023.

22. Messias MCTB, Menegatto MF, Prado ACC, Santos BR, Guimarães MFM. Uso popular de plantas medicinais e perfil socioeconômico dos usuários: um estudo em área urbana em Ouro Preto, MG, Brasil. Rev. Bras. Plantas Med [Internet] 2015. [acesso em 05 nov. 2019]; 17(1). Disponível em: http://dx.doi. org/10.1590/1983-084X/12 139.

23. Nascimento W de MC, Melo OF, Silva IF, Souza FL de. Plantas medicinais e sua utilização pelas comunidades do município de Sobral, Ceará. SANARE [Internet] 2013. [acesso em 05 nov. 2019]; 12(1). Disponível em: https://sanare.emnuvens.com.br/sanare/article/view/328.

24. Arentz S, Smith CA, Abbott J, Fahey P, Cheema BS, Bensoussan A. Combined Lifestyle and Herbal Medicine in Overweight Women with Polycystic Ovary Syndrome (PCOS): a randomized controlled trial. Phytother Res [Internet]. 2017. [acesso em 05 nov. 2019]; 31(9). Disponível em: http://dx.doi.org/10.1002/ ptr.5858.

25. Adnan M, Tariq A, Mussarat S, Begum S, Abdeisalam NM, Ullah R. Ethnogynaecological Assessment of Medicinal Plants in Pashtun's Tribal Society. Biomed Research International. [Internet] 2015. [acesso em 05 nov. 2019]; (ID196475). Disponível em: http://dx.doi.org/10.1155/2015/196475.

26. Haj-Husein I, Tukan S, Alkazaleh F. The effect of marjoram (O riganum majorana) tea on the hormonal profile of women with polycystic ovary syndrome: a randomised controlled pilot study. J Hum Nutr Diet [Internet] 2015. [acesso em 05 nov. 2019]; 29(1). Disponível em: http://dx.doi.org/10.1111/jhn.12290. 
Recebido: 15/01/2020

Finalizado: 21/07/2020

Editora associada: Tatiane Herreira Trigueiro

Autor Correspondente:

Maria Charlianne de Lima Pereira Silva

Instituto Centro de Ensino Tecnológico

R. Lauro Pontes, 56 - 62785-000 - Acarape, CE, Brasil

E-mail: mcharlianne@yahoo.com.br

Contribuição dos autores:

Contribuições substanciais para a concepção ou desenho do estudo; ou a aquisição, análise ou interpretação de dados do estudo - MCLPS, JCAA, LBS, ECC, OROP, NSM, FJML

Elaboração e revisão crítica do conteúdo intelectual do estudo - MCLPS, JCAA, ECC, OROP, NSM, FJML

Aprovação da versão final do estudo a ser publicado - LBS, ECC

Responsável por todos os aspectos do estudo, assegurando as questões de precisão ou integridade de qualquer parte do estudo - MCLPS

\section{(c) (1)}

Este obra está licenciado com uma Licença Creative Commons Atribuição 4.0 Internacional. 